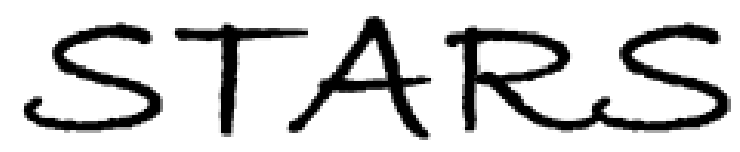

University of Central Florida

STARS

$1-1-2015$

\title{
Gold-doped graphene: A highly stable and active electrocatalysts for the oxygen reduction reaction
}

\author{
Sergey Stolbov \\ University of Central Florida \\ Marisol Alcántara Ortigoza \\ University of Central Florida
}

Find similar works at: https://stars.library.ucf.edu/facultybib2010 University of Central Florida Libraries http://library.ucf.edu

This Article is brought to you for free and open access by the Faculty Bibliography at STARS. It has been accepted for inclusion in Faculty Bibliography 2010 s by an authorized administrator of STARS. For more information, please contactSTARS@ucf.edu.

\section{Recommended Citation}

Stolbov, Sergey and Ortigoza, Marisol Alcántara, "Gold-doped graphene: A highly stable and active electrocatalysts for the oxygen reduction reaction" (2015). Faculty Bibliography 2010s. 6812. https://stars.library.ucf.edu/facultybib2010/6812

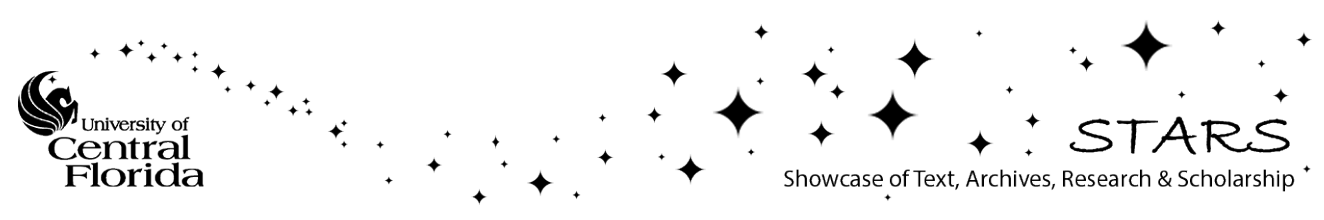




\section{Gold-doped graphene: A highly stable and active electrocatalysts for the oxygen reduction reaction}

Cite as: J. Chem. Phys. 142, 154703 (2015); https://doi.org/10.1063/1.4917468

Submitted: 15 December 2014 . Accepted: 01 April 2015 . Published Online: 17 April 2015

Sergey Stolbov, and Marisol Alcántara Ortigoza
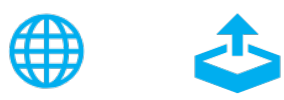

\section{ARTICLES YOU MAY BE INTERESTED IN}

Gold in graphene: In-plane adsorption and diffusion

Applied Physics Letters 94, 043106 (2009); https://doi.org/10.1063/1.3075216

Trapping of metal atoms in the defects on graphene

The Journal of Chemical Physics 135, 224704 (2011); https://doi.org/10.1063/1.3666849

Factors controlling the energetics of the oxygen reduction reaction on the Pd-Co electrocatalysts: Insight from first principles

The Journal of Chemical Physics 135, 134702 (2011); https://doi.org/10.1063/1.3643714

Where in the world is AIP Publishing? Find out where we are exhibiting next 


\title{
Gold-doped graphene: A highly stable and active electrocatalysts for the oxygen reduction reaction
}

\author{
Sergey Stolbov ${ }^{\mathrm{a})}$ and Marisol Alcántara Ortigoza ${ }^{\mathrm{b})}$ \\ Department of Physics, University of Central Florida, Orlando, Florida 32816-2385, USA
}

(Received 15 December 2014; accepted 1 April 2015; published online 17 April 2015)

\begin{abstract}
In addressing the growing need of renewable and sustainable energy resources, hydrogen-fuel-cells stand as one of the most promising routes to transform the current energy paradigm into one that integrally fulfills environmental sustainability. Nevertheless, accomplishing this technology at a large scale demands to surpass the efficiency and enhance the cost-effectiveness of platinum-based cathodes, which catalyze the oxygen reduction reaction (ORR). In this work, our first-principles calculations show that $\mathrm{Au}$ atoms incorporated into graphene di-vacancies form a highly stable and cost-effective electrocatalyst that is, at the same time, as or more (dependently of the dopant concentration) active toward ORR than the best-known Pt-based electrocatalysts. We reveal that partial passivation of defected-graphene by gold atoms reduces the reactivity of $\mathrm{C}$ dangling bonds and increases that of $\mathrm{Au}$, thus optimizing them for catalyzing the ORR and yielding a system of high thermodynamic and electrochemical stabilities. We also demonstrate that the linear relation among the binding energies of the reaction intermediates assumed in computational high-throughput material screening does not hold, at least for this non-purely transition-metal material. We expect Au-doped graphene to finally overcome the cathode-related challenge hindering the realization of hydrogen-fuel cells as the leading means of powering transportation and portable devices. () 2015 AIP Publishing LLC. [http://dx.doi.org/10.1063/1.4917468]
\end{abstract}

\section{INTRODUCTION}

Hydrogen fuel cells, devices materializing the clean conversion of chemical energy of hydrogen into electric energy, are already proven means of powering transportation and portable devices. Nonetheless, this clean technology has not boomed to the desired level in large part because of the cost and efficiency of the Pt (or Pt-based) cathode catalysts that enable the electrocatalytic mechanism at the heart of the hydrogen-fuel-cell operation: the oxygen reduction reaction (ORR).

An enormous effort has been made in searching for more efficient and cost-effective ORR catalysts. The main direction so far has been only that of reducing the cost of the catalysts by decreasing the load of Pt or Pt-group elements in them. The most successful attempts involve catalysts in the form of core-shell nanoparticles with Pt shell and Pt-Fe, Pt-Co, Pd-Fe, and Ir-Co cores. ${ }^{1-6}$ A clear disadvantage of such designs is that they still contain a large (even dominating) fraction of the most expensive and scarce Pt or Pt-group elements. In fact, to our knowledge, there is only one attempt to design pure transition-metal but Pt-free ORR catalysts. ${ }^{7}$ And even so, the dream of surpassing the catalytic performance of $\mathrm{Pt}$ has not been achieved so far, despite decades of intensive research.

The maximum onset potential $U_{0}$ for Pt electrocatalysts with respect to the standard hydrogen electrode (SHE), which

\footnotetext{
a) Author to whom correspondence should be addressed. Electronic mail: Sergey.Stolbov@ucf.edu

b) Electronic address: Marisol.AlcantaraOrtigoza@ucf.edu
}

is defined as that measured at a negligible low ORR current (and thus negligible power), is only $U_{0}=0.9 \mathrm{eV}$ (SHE); that is, $73 \%$ of the ideal value $(1.23 \mathrm{~V})$. In principle, one could envision to eventually overcome this limit by high-throughput screening of endless combinations of elements in alloys and/or multilayers designs. Nevertheless, the very nature of the hydrogen-fuel-cell operation inherently poses an almost final and extremely narrow restriction on the materials that can serve as ORR electrocatalysts. Namely, most elements of the periodic table dissolve while in contact with the surrounding medium created by the very reactants, and even more when they are under the action of the potential produced by the fuel cell. In fact, only three elements have in the bulk a nominal dissolution potential $U_{\text {diss }}$ higher than the operating potential (that created when the power of the fuel cell is maximum) of what would be an efficient fuel cell ( $\sim 0.8-1.0 \mathrm{~V}$ (SHE)): $U_{\text {diss }}$ of $\mathrm{Ir}, \mathrm{Pt}$, and $\mathrm{Au}$ is $1.16,1.18$, and $1.5 \mathrm{~V}$ (SHE), respectively. ${ }^{8}$ Yet, in practice the problem is actually more severe. For example, even though the nominal dissolution potential of Pt is $\sim 1.18 \mathrm{~V}$ (SHE) in acidic medium $(\mathrm{pH}=0)$, the metal's dissolution already sets in at an electrode potential of $0.65 \mathrm{~V},{ }^{9}$ which is much lower than the operating potential of an efficient fuel cell.

Clearly, the above represents one more drawback of $\mathrm{Pt}$ urging us to direct our attention toward more electrochemically stable materials. The quest is not at all hopeless because, on the one hand, (1) $U_{\text {diss }}$ can be increased by means of increasing the bond-strength of the catalytically active metal atoms. ${ }^{10}$ Regrettably, the efforts for reducing the load of Pt by depositing Pt monolayers on other transition-metal substrates have yield a negligible enhancement of the electrochemical 
stability of Pt, because the binding strength of Pt to those substrates is marginally increased. ${ }^{11}$ (2) On the other hand, bulk gold is by far and so far the most stable metal against dissolution: it has $U_{\text {diss }} \sim 1.5 \mathrm{~V}$ (SHE). Naturally, being bulk $\mathrm{Au}$ the noblest metal, the challenge has been to activate it chemically toward ORR. One way to make Au more reactive is, of course, to use it in the form of small (sub-nanometer) particles. ${ }^{12}$ Nevertheless, reducing the particle size causes a drastic decrease in the bond strength of the particle' surface atoms and thus in their stability in the reaction environment. ${ }^{9,13}$ Another way to activate gold is to bind Au atoms to some reactive material such that the electronic hybridization giving rise to the bond may lead to a redistribution of the $\mathrm{Au}$ $d$-electronic states that, in turn, significantly increase $\mathrm{Au}$ activity toward ORR at the level of that of $\mathrm{Pt}$ or even higher. ${ }^{7}$ Concerning studies beyond transition-metal electrocatalysts, there have been a number of attempts to explore the graphene-based materials as ORR electrocatalysts. ${ }^{14-26}$ However, in most cases graphene has been used just as a substrate for small metal or metal oxide clusters. ${ }^{14-18}$ As such, the graphene-metal interaction is thus not critical for the electrocatalytic properties. The authors of Refs. 19-25 did consider systems with actual doping of graphene in which the electronic states of the dopants happened to be modified due to a strong dopant-graphene bonding. Computational studies of ORR on N-doped graphene, ${ }^{19}$ for example, show that this doping facilitates binding of the ORR intermediates to the system, whereas pure graphene does not exhibit such activity. In fact, combined experimental and computational studies of $\mathrm{B}-, \mathrm{N}-, \mathrm{O}-, \mathrm{P}-$, and S-doped graphene ${ }^{20}$ showed that while S-doping does not facilitate ORR whatsoever boron doping gives the best improvement of the ORR activity. Nevertheless, even the most active B-doped catalyst has an activity toward ORR much lower than that of Pt. Furthermore, the experiment and calculations for such system were performed in an alkaline solution. Yet alkaline solutions are not relevant to the proton exchange membrane fuel cells, which are the focus of this work. Yet another study reported that the reduced graphene oxide doped with $\mathrm{FeN}_{4}$ complexes exhibits a high activity toward ORR at a low electrode potential. ${ }^{21}$ However, the onset potential of this material appears to be significantly lower than that of Pt. Furthermore, it has a low stability in the reaction environment: at an electrode potential of $0.5 \mathrm{~V}$, the ORR current remains constant over $70 \mathrm{~h}$, but at $0.75 \mathrm{~V}$, the ORR activity decreases in $24 \mathrm{~h}$ to $45 \%$ of the initial value.

The above works show that graphene doping is a promising direction for finding efficient ORR catalysts. However, the so far obtained materials either have a relatively low activity toward ORR or are not stable in the reaction environment. In this work, guided by the many attempts and the variety of systems investigated to overcome this challenging problem, we explore a somewhat different approach that is neither activating a metal nor a dopant in graphene, but instead using a metal to dope, passivize and thus activate the very same graphene. We propose that the incorporation of $\mathrm{Au}$ atoms into the well-known carbon vacancies created in graphene should yield simultaneously a high ORR activity and electrochemical stability to levels never previously achieved simultaneously for carbon-based materials or transition-metal-based materials.
Our design responds to two main guidelines: (1) while pristine graphene is not active at all, vacancies are too active. Thus, incorporating a metal atom into the vacancy will only partially saturate the $\mathrm{C}$ dangling bonds and reduce the reactivity of these sites. (2) The electronic states redistribution may in principle make the metal dopant atoms active toward ORR but, more importantly, the expected strong metal-C hybridization is targeted to avoid that the metal dissolves in the reaction environment and, in this regard, Au is the best choice for its already intrinsic electrochemical stability.

A key factor guaranteeing the feasibility of this design is that Au-atom dopants are incorporated into one of the most thermodynamically stable carbon vacancies created in graphene: the 5-8-5 di-vacancy (DV). Indeed, observations and first-principles calculations show that these DVs attract and trap metal atoms. ${ }^{27-29}$ Furthermore, it has been reported that $\mathrm{Au}$ is strongly bound to the DV and its diffusion barriers from that are high $(\sim 4 \mathrm{eV})$, which ensures stability of the proposed system. ${ }^{30,31}$ Incidentally, this phenomenon is a sign of the high reactivity of the under-coordinated carbon atoms at the edge of the vacancy and the strong bonds they make with $\mathrm{Au}$ and $\mathrm{Pt}$, all of which we shall elucidate through an analysis of their electronic structure. Our $a b$-initio calculations will furthermore provide evidence of the remarkable thermodynamic and electrochemical stabilities of the proposed system. Clearly, other defects may be present in graphene, such as mono-vacancies and free edges. However, these rarer defects can also trap Au atoms and thus be passivated. The bottom line is that, although investigating the catalytic activity of other Au-passivated defects is beyond the scope of the present study, our work already hints that gold passivation may prevent the corrosion or dissolution of graphene by the acidic media of hydrogen fuel cell cathode.

It must be mentioned that an additional merit of the efforts directed to find better electrocatalysts for the cathode of hydrogen fuel cells is the simulation advancements as means to predetermine the electrocatalytic activity of a given system toward ORR. One of the most important achievements is the sound establishment of the link between the ORR activity of a surface and the free energy of the ORR intermediates adsorbed on the surface..$^{32}$ Namely, the ability of such correlation to reproduce and/or predict the experimental onset potential $U_{0}$ has demonstrated that computational simulations have an excellent predictive power to evaluate ORR activity. ${ }^{32}$ This is true not only for transition-metal catalysts ${ }^{7,33-36}$ but has also been proved for defected graphene doped with $\mathrm{B}, \mathrm{N}, \mathrm{O}$, $\mathrm{P}$, and $\mathrm{S}^{20}$ And yet, we shall disprove and call for caution in applying an oversimplifying notion postulating that the binding energy $E_{B}$ of the three intermediates of the reaction is in general linearly correlated: $E_{B}(O H)=a E_{B}(O)+b$ and $E_{B}(O O H)=c E_{B}(O)+d .^{5,37}$ Clearly, in such scenario $E_{B}(O)$ alone becomes the absolute descriptor for surface activity toward ORR. The existence of a linear dependence between these energies has certainly been proved for the Pt- and Pd-based catalysts. However, such linear dependence may not necessarily hold in general since each of the reaction intermediates may perturb the catalysts electronically and/or geometrically at a quite different degree, as we shall discuss later. 


\section{ORR THERMODYNAMICS MODEL AND COMPUTATIONAL DETAILS}

To evaluate the activity of an electrocatalyst toward ORR, we apply the well-known approximation in which the ORR onset potential of a surface is entirely determined by the free energy $G$ of each of the ORR intermediates adsorbed to the surface. ${ }^{32}$ These values are used to build ORR free-energy diagrams for arbitrary electrode potentials $U$, which in turn let us determine the onset potential $U_{0}$ of the catalysts for ORR.

For highly active ORR catalysts, the dissociation of oxygen molecules adsorbed on the surface is not favorable and the reaction is expected to proceed through the following steps:

$$
\begin{gathered}
\mathrm{O}_{2}+{ }^{*} \rightarrow \mathrm{O}_{2}{ }^{*}, \\
\mathrm{O}_{2}{ }^{*}+\left(\mathrm{H}^{+}+\mathrm{e}^{-}\right) \rightarrow{ }^{*} \mathrm{OOH}, \\
{ }^{*} \mathrm{OOH}+\left(\mathrm{H}^{+}+\mathrm{e}^{-}\right) \rightarrow \mathrm{H}_{2} \mathrm{O}+\mathrm{O}^{*}, \\
\mathrm{O}^{*}+\left(\mathrm{H}^{+}+\mathrm{e}^{-}\right) \rightarrow \mathrm{HO}^{*}, \\
\mathrm{HO}^{*}+\left(\mathrm{H}^{+}+\mathrm{e}^{-}\right) \rightarrow \mathrm{H}_{2} \mathrm{O}+{ }^{*},
\end{gathered}
$$

where "*” denotes an adsorption site on the catalyst's surface. $\Delta G$ for each intermediate state is calculated as the difference between $G$ of that intermediate state and that of the final state of the reaction: the clean catalyst surface plus $2 \mathrm{H}_{2} \mathrm{O}$ in gas phase. Note that $\mathrm{H}_{2} \mathrm{O}$ binding to surfaces is neglected in the diagrams. Customarily, the influence of the binding strength of water to the electrocatalyst is neglected because the $\mathrm{H}_{2} \mathrm{O}$ binding is very weak $(\sim 0.20 \mathrm{eV})$ and, importantly, varies from one surface to the other by no more than $30 \mathrm{meV}$.

Since the reaction steps involve charge transfer, the relevant contribution to the free energy $G$ of that intermediate state due to the transferred protons across the potential difference is determined as follows: $G_{U}=-n e U$, where $U$ is the electrode potential (e.g., the potential difference between anode and cathode, which is treated as a variable) and $n$ is the number of protons that will be transferred in the course of the reaction from that intermediate state to the final state. Consequently, for each ORR intermediate state, the free energy $\Delta G$ can be defined as follows: ${ }^{32}$

$$
\Delta G=\Delta E+\Delta Z P E-T \Delta S+\Delta G_{U} .
$$

Here, $\Delta E$ is the ground-state internal energy of the catalyst surface adsorbed with an intermediate and is obtained from the density functional theory (DFT) total-energy calculations described below. $\triangle Z P E$ is the zero-point energy correction and is determined from DFT-based calculations of the vibrational frequencies of each adsorbate using the finite-differences method. In the case of transition-metal-based catalysts, the vibration frequencies of the ORR intermediates are calculated with "frozen" substrate because the mass of substrate atoms is much larger than those of the intermediates, and the corresponding vibrational modes are essentially decoupled. ${ }^{7,36}$ In the Au-graphene case, however, this approximation may not be sufficient because the masses of $\mathrm{C}, \mathrm{O}$, and even $\mathrm{H}$ are comparable. To test this hypothesis, we calculate the ZPE of adsorbed $\mathrm{O}, \mathrm{OH}$, and $\mathrm{OOH}$ in the following three manners: (a) the graphene sheet frozen - only the adsorbate is free to vibrate; (b) the adsorbate and its neighboring $\mathrm{C}$ atom are allowed to vibrate, while the rest of the substrate is frozen; (c) the adsorbate, its nearest neighbor $\mathrm{C}$ atoms, and next neighbor $\mathrm{C}$ atoms are allowed to vibrate, while the rest of the substrate is frozen. We found that values of ZPE obtained for approximations (b) and (c) are close to each other, while ZPE is noticeably larger for case (a) than for cases (b) and (c). For example, for $\mathrm{OOH}$ adsorbed on Au-graphene, we have $\mathrm{ZPE}=457 \mathrm{meV}, 442 \mathrm{meV}$, and $439 \mathrm{meV}$ for the (a), (b), and (c) approximations, respectively. These results assured us that using approximation (c) provides results accurate enough for the energy scale under consideration.

The entropic contribution $T \Delta S$ is approximated by the gas-phase entropy of the reactants or intermediates, which is taken from the CODATA tables ${ }^{38}$ (for adsorbed species, translational and rotational contributions are subtracted).

To include contributions of transferred protons to the free energy, the chemical potential of $\left(\mathrm{H}^{+}+\mathrm{e}^{-}\right)$is approximated by one-half of the chemical potential of the $\mathrm{H}_{2}$ molecule. ${ }^{32}$ After some algebraic manipulations, the total energy of a catalyst adsorbed by an intermediate with respect to the total energy of the final state (see above) can be expressed through the binding energies $E_{B}$ of the intermediates. ${ }^{36}$ For example, for the states $\mathrm{O}^{*}$ and $\mathrm{OH}^{*}$, described by Eqs. (4) and (5), such energy difference can be expressed as

$$
\begin{aligned}
\Delta E(O)= & E_{t o t}\left(H_{2}\right)+E_{t o t}(O)-E_{t o t}\left(H_{2} O\right)-E_{B}\left(O^{*}\right), \\
\Delta E(O H)= & \frac{1}{2} E_{t o t}\left(H_{2}\right) \\
& +E_{t o t}(O H)-E_{t o t}\left(H_{2} O\right)-E_{B}\left(O H^{*}\right) .
\end{aligned}
$$

The first three terms on the right side of Eqs. (7) and (8) represent the total energy of molecules in gas phase. Therefore, in these equations, only the $E_{B}$ terms depend on the properties of the catalyst surface.

The calculation of the binding energy of the various intermediates is performed in the presence of four water molecules per supercell in the vicinity of each intermediate in order to model solvent effects. ${ }^{7,32,36}$

All our DFT-based calculations have been performed using the VASP5.2 code $^{39}$ with projector augmented wave

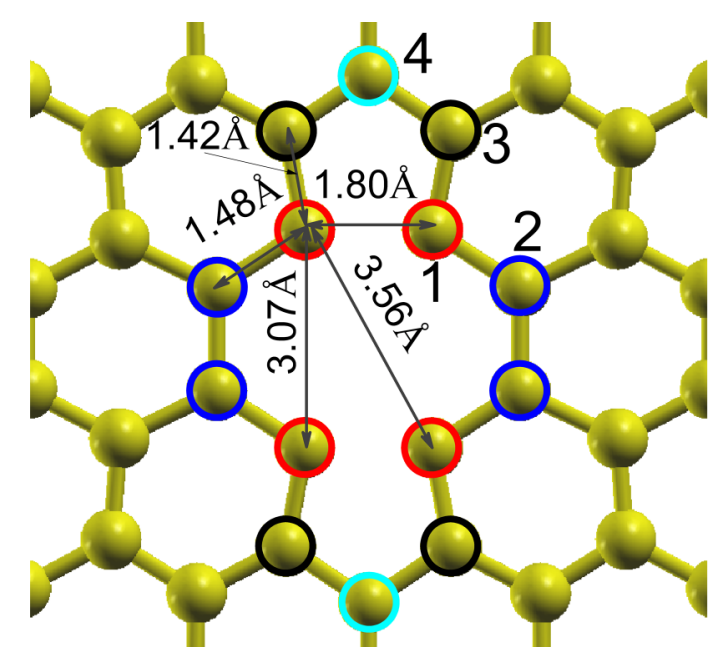

FIG. 1. The relaxed structure of a graphene sheet with a 5-8-5-DV in the $6 \times 6$ structure. As shown by the bond-lengths, type 1 atoms (C1) have essentially coordination 2, whereas type 2-4 atoms (C2-C4) have coordination 3. 
potentials $^{40}$ and with the Perdew-Burke-Ernzerhof (PBE) parameterization for the exchange and correlation functional. ${ }^{41}$ In order to maintain periodicity, we use a supercell that includes the defected (with one 5-8-5 DV) graphene sheet with $4 \times 4$ and $6 \times 6$ in-plane periodicities and a $\sim 14 \AA$ vacuum layer. The $(4 \times 4 \times 1)$ and $(3 \times 3 \times 1) k$-point samplings in Brillouin zone used in this work for the $4 \times 4$ and $6 \times 6$ structures, respectively, provided sufficient accuracy for the characteristics obtained by integration in the reciprocal space. The cutoff energies of $400 \mathrm{eV}$ and $600 \mathrm{eV}$ were used for the plane wave expansion of wave functions and charge density, respectively. The atomic relaxation was carried out until the force acting on each atom and each direction does not exceed $0.01 \mathrm{eV} / \AA$.

To characterize the strength of the bonding between an intermediate $X$ and the Au-graphene catalyst, we used the binding energy defined as follows:

$$
\begin{aligned}
E_{B}(X)= & E_{t o t}(\text { Au-graphene }) \\
& +E_{t o t}(X) E_{t o t}(X / \text { Au-graphene }),
\end{aligned}
$$

where the three $E_{t o t}$ terms denote the total energy per supercell calculated for the clean doped graphene sheet; the isolated (and neutral) intermediate $X=\mathrm{O}, \mathrm{OH}, \mathrm{OOH}$; and $X$ adsorbed on the Au-doped graphene sheet, respectively. Since the total energy of a stable system is negative, $E_{B}(O H)$ is defined positive provided that the adsorption of a specie on the catalysts is energetically favorable.

The Xcrysden software ${ }^{42}$ was used to plot geometric structures of the systems under consideration.

\section{RESULTS AND DISCUSSION}

\section{A. Electronic structure and stability of Au-doped graphene}

In this work, we model the incorporation of Au atoms into carbon DVs of the 5-8-5 symmetry, not only because this DV is much more stable thermodynamically than singlevacancy defects ${ }^{29}$ but also because it yields carbon atoms at the edge of the vacancy of high reactivity, the origin of which we shall reveal in the following. The calculations have been performed for two vacancy concentrations: (a) one vacancy per $4 \times 4$ supercell and (b) one vacancy per $6 \times 6$ supercell in order to draw conclusions that are not contingent on the proximity between DVs. The optimized (relaxed) structure of a 5-8-5 DV in a graphene sheet for the $6 \times 6$ periodicity is illustrated in Figure 1. First of all, despite two carbon atoms are missing causing some atoms at the edge of the vacancy to have dangling bonds, for none of the two concentrations (the defected graphene sheet with $4 \times 4$ and $6 \times 6$ periodicities) the carbon atoms of graphene become magnetic, in agreement with previous calculations. ${ }^{43,44}$ One can see in Fig. 1 that there are four non-equivalent types of $\mathrm{C}$ atoms defining the 5-8-5 DV: type 1 (four $\mathrm{C} 1$ atoms), type 2 (four $\mathrm{C} 2$ atoms), type 3 (four $\mathrm{C} 3$ atoms), and type 4 (two $\mathrm{C} 4$ atoms). Types $2-4$, however, are fully coordinated (coordination 3 ) through three covalent bonds of approximately the typical $\mathrm{C}-\mathrm{C}$ bond length in graphene, $1.42 \AA$. In contrast, type 1 atoms in the $6 \times 6$ supercell have two neighbors at $1.42 \AA$ and $1.48 \AA$, respectively, and one at $1.80 \AA$, which is in a good agreement with results obtained by other authors. ${ }^{45}$ Interestingly, for the $4 \times 4$ structure the latter distance is larger $(1.99 \AA)$, which may reflect a noticeable interaction between vacancies. We calculated the DV formation energy using the definition of this quantity proposed in Ref. 30 and found it to be $8.252 \mathrm{eV}$ for the $4 \times 4$ structure and $7.929 \mathrm{eV}$ for $6 \times 6$, which is in good agreement with results obtained in Ref. $30(\sim 8 \mathrm{eV})$. Nevertheless, both distances are large enough so that no relevant covalent bond exists between the two $\mathrm{C} 1$ carbon atoms. Thus, one expects the four two-coordinated (type 1) atoms in each 5-8-5 DV to be those chemically active. This is confirmed by comparing the projected local densities of electronic states (PLDOSs) of carbon in pristine graphene and that of the 5-8-5 DV edge $\mathrm{C} 1$ atoms calculated for the $4 \times 4$ structure. Naturally, striking deviations from the PLDOS of pristine-graphene atoms are seen only for the twocoordinated atoms of the 5-8-5 DV, whereas the PLDOS of three-coordinated atoms does not differ significantly from that of the atoms of pristine graphene. Thus in the following, we shall focus our attention only on atoms type 1 .

As seen from Figure 2, the PLDOS of the two-coordinated atoms is in general significantly enhanced at energies closer to the Fermi level, $\mathrm{E}_{\mathrm{F}}$, with respect to that of pristine graphene. This indicates that these atoms are indeed those making the 5-8-5 DV in graphene reactive. Namely, while in pristine graphene the occupied and unoccupied in-plane

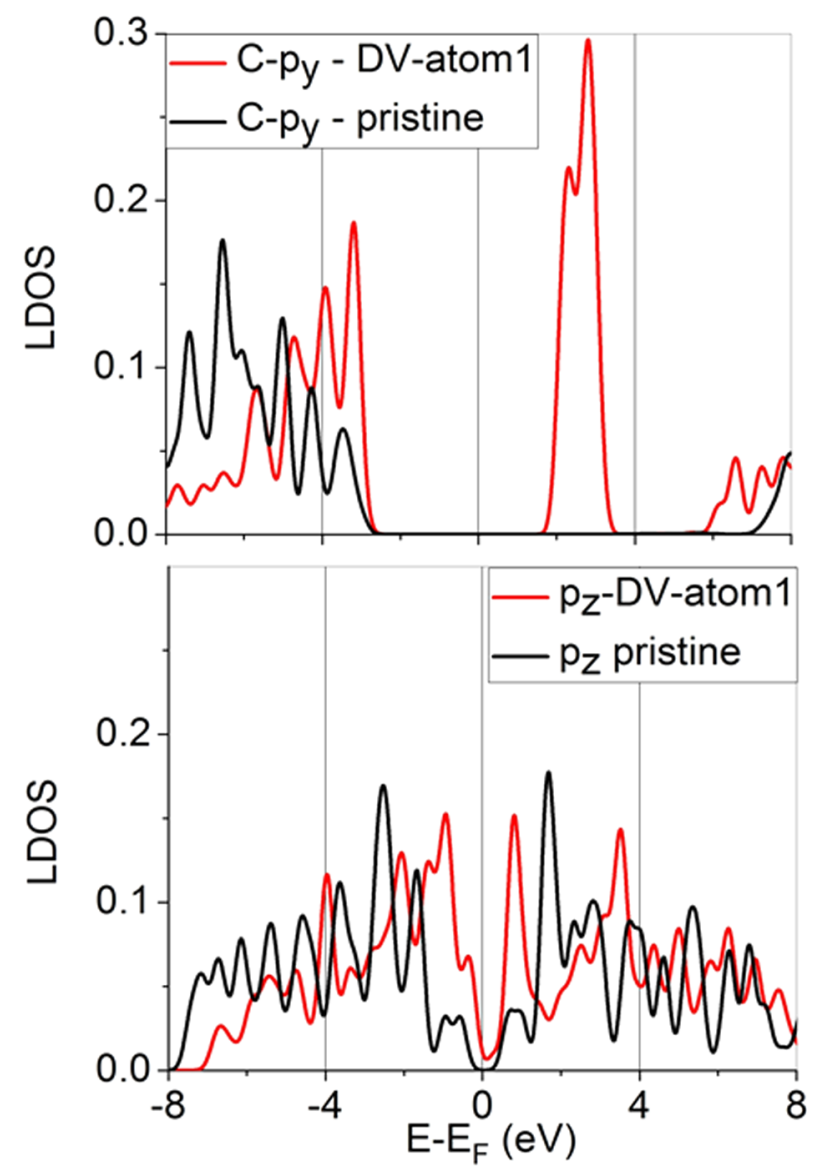

FIG. 2. Projected local densities of electronic states calculated for a C-atom in pristine graphene and the atom " $\mathrm{C} 1$ " in graphene with a 5-8-5 DV in the $4 \times 4$ structure. 
$p$-states (e.g., $p_{y}$ ) of $\mathrm{C}$ are separated by a markedly large gap of $\sim 9 \mathrm{eV}-$ reflecting the strong $\mathrm{C}-\mathrm{C}$ covalent bonding through $s p^{2}$-hybridized states, this energy gap in the PLDOS of two coordinated atoms of the 5-8-5 DV is reduced to less than $50 \%$ of this value, i.e., only $\sim 4 \mathrm{eV}$, as shown in Fig. 2 (top). More importantly, while pristine graphene atoms have a low density of states in the vicinity of $\mathrm{E}_{\mathrm{F}}$ - even beyond the region corresponding to the well-known linear dispersion of the $p_{z}$ bands at $\mathrm{E}_{\mathrm{F}}$, the two-coordinated atoms of the 5-8-5 DV acquire a striking enhancement in that region for both occupied and unoccupied states (in the interval $[-1,1] \mathrm{eV}$ with respect to $E_{F}$ ), as shown in Fig. 2 (bottom). Moreover, the separation between the sharpest peaks of the occupied and unoccupied $p_{z}$-PLDOSs of the two-coordinated $\mathrm{C}$ atoms is also strongly reduced with respect to that of pristine graphene: from $\sim 4 \mathrm{eV}$ to less than $2 \mathrm{eV}$.

Clearly, the redistribution of PLDOS shown above suits for a much stronger hybridization between the electronic states of a metal dopant and the $p$-states of the two-coordinated C atoms than between the states of a metal atom and those of a carbon atom in pristine graphene. ${ }^{46,47}$ Importantly as well, the size of the Au atom matches the dimension of the 5-8-5 DV (regardless of the concentration). In particular, the distances between type 1 atoms suit for making four $\mathrm{Au}-\mathrm{C}$ bonds in this configuration, as suggested by the distances indicated in Fig. 1.

As expected, a gold monomer finds indeed its lowest total energy at the center of the 5-8-5 DV, position at which it makes four bonds with $\mathrm{C}$ atoms, one with each of the four two-coordinated $\mathrm{C} 1$ atoms, as shown in Figure 3. The resulting $\mathrm{Au}-\mathrm{C}$ bonds are very strong. In agreement with earlier calculations, ${ }^{31}$ our results indicate that the binding energy of the $\mathrm{Au}$ adatom in the 5-8-5 DV (with respect to a single isolated $\mathrm{Au}$ atom) in such configuration is $\mathrm{E}_{\mathrm{B}}(\mathrm{Au})=4.487 \mathrm{eV}$ for the $4 \times 4$ structure and $4.240 \mathrm{eV}$ for the $(6 \times 6)$ structure. PBE is known to underestimate the cohesive energy of bulk $\mathrm{Au}$, so one can expect that the actual $\mathrm{E}_{\mathrm{B}}(\mathrm{Au})$ may also be larger than predicted by DFT calculations. But even if we consider that the actual binding energy of the adatom to the DV vacancy is at least $4.28 \mathrm{eV}$, such binding energy would be at least $0.43-0.67 \mathrm{eV}$ higher than experimental cohesive energy of bulk Au.

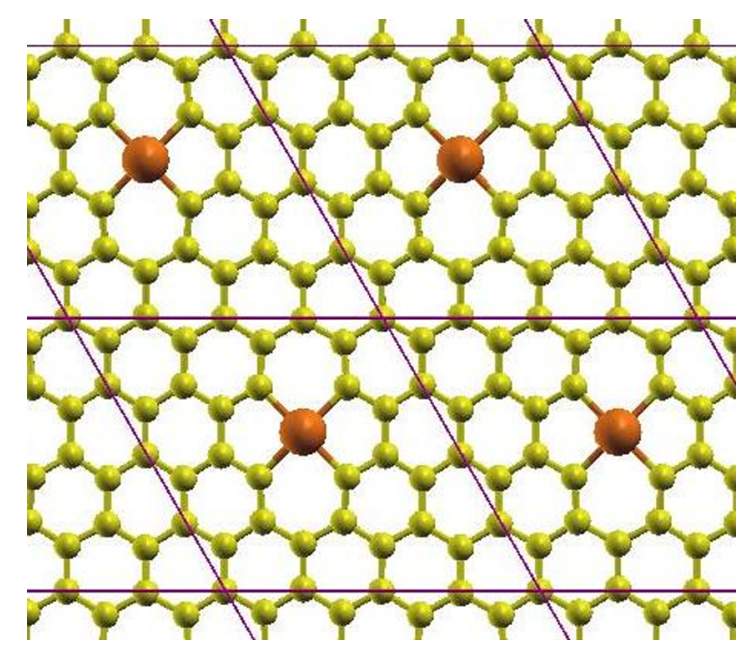

FIG. 3. Geometric structure of the Au-doped graphene in the $4 \times 4$ structure. Small yellow and large orange balls denote $\mathrm{C}$ and $\mathrm{Au}$, respectively.
Thus, the results above show that there are two more virtues of passivating carbon dangling bonds via Au doping, in particular. Upon strong binding to the DVs, the Au atoms (1) become stabilized thermodynamically and (2) increase their $U_{\text {diss }}$ (see Refs. 10 and 13). Thermodynamic stability and its implications for the preparation of the designed samples are critical aspects of computational design because sample preparation and preservation are each challenges in themselves. In the present case, the strikingly strong binding energy of the Au dopant is ensuring that, upon sub-monolayer Au deposition on a defected graphene sheet (with 5-8-5 DVs), some $\mathrm{Au}$ atoms will certainly take the sites shown in Fig. 3 rather than precipitating as Au particles. These results are in line with available experimental results. Namely, it has been reported that in the course of preparation of a system like this, metal (Au or Pt) clusters may appear around vacancies but have high diffusivity and are unstable, because its atoms readily take the edge sites of the other vacancies when available. ${ }^{48}$ Our results in addition show that the basis of the single-atom growth mechanism seeded in the dopant is not particularly favorable. For example, we find that a dimer near the 5-8-5 vacancy dissociates with no energy activation barrier. Upon relaxation, one of the Au atoms forming the dimer disconnects from the other and becomes a dopant of the defected graphene (see Fig. 3), whereas the other makes a bond with only one neighboring $\mathrm{C}$ atom, as shown in Figure 4. This bond with $\mathrm{C}$ is weak; the corresponding binding energy, $\mathrm{E}_{\mathrm{B}}(\mathrm{Au})=1.209 \mathrm{eV}$, which is dramatically smaller than the binding energy of the dopant and comparable to a Au dimer formation energy in vacuum or on pristine graphene.

Similarly, if a planar (most stable) four-atom Au cluster is placed in the vicinity of the 5-8-5 DV (see left panel of Fig. 5), it becomes unstable. In the ground state of such system, it decomposes with no activation energy barrier into a dopant $\mathrm{Au}$ atom and a weakly adsorbed $\mathrm{Au}$ trimer. The Au trimer is bound to the $\mathrm{Au}$-doped graphene via a single $\mathrm{Au}-\mathrm{C}$ bond made with a $\mathrm{C}$ atom that is neighbor of the Au dopant, as shown in the right panel of Figure 5. But again, the binding energy of the trimer is only $1.821 \mathrm{eV}$, which is also much

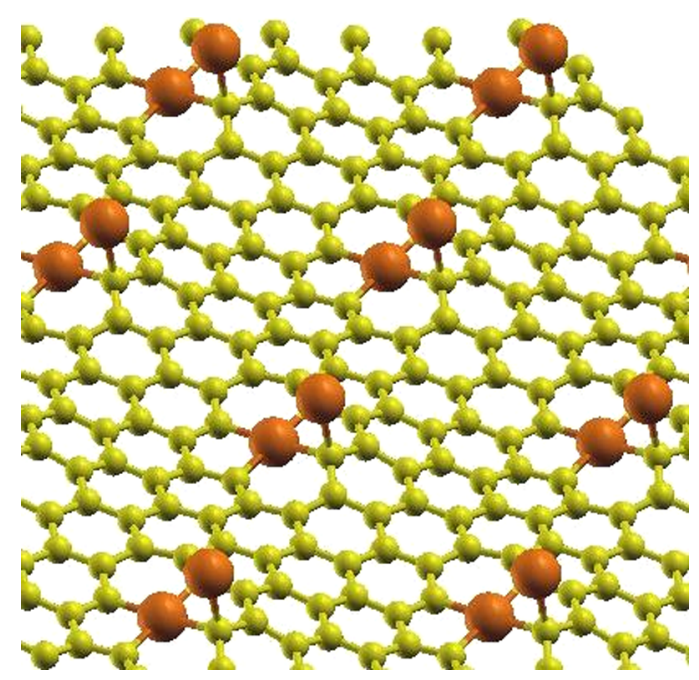

FIG. 4. Optimized configuration of a dissolved dimer because of a 5-8-5 DV in the graphene $4 \times 4$ structure. Small and large balls denote $\mathrm{C}$ and $\mathrm{Au}$ atoms, respectively. 

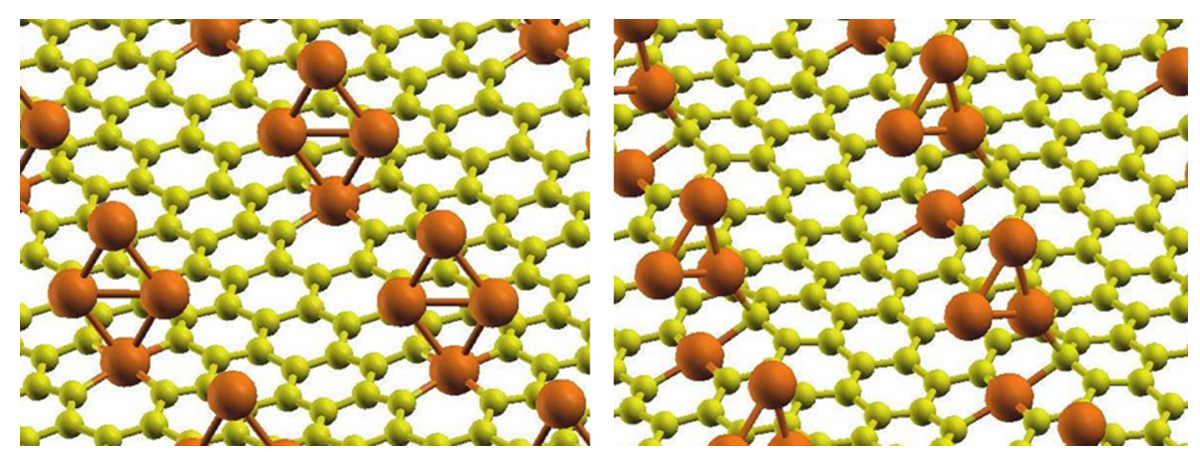

FIG. 5. Structural optimization of 4 Au-atom planar clusters near 5-8-5 DVs in the $4 \times 4$ graphene structure. Left panel: initial configuration. Right panel: optimized configuration. Small and large balls represent $\mathrm{C}$ and $\mathrm{Au}$ atoms, respectively. smaller than the binding energy of the Au dopant. This means that the binding energy of anchored $\mathrm{Au}$ atoms to the dopants or its carbon neighbors is relatively weak ${ }^{48}$ with respect to the binding energy of the Au dopant.

Therefore, in terms of realizing a clean sample, the latter results indicate that such particles can be removed without breaking the bonds made by the Au-dopant to the defected graphene. Specifically, small Au particles can be dissolved by applying the electrochemical method described in Refs. 49 and 50, which guarantees the integrity of the $\mathrm{Au}-\mathrm{C}$ bonds made by the Au dopant because the formation energy of small Au particles is much smaller than the cohesive energy of bulk $\mathrm{Au}, 3.814 \mathrm{eV}$, and thus, their $U_{\text {diss }}$ is significantly smaller. ${ }^{10,13}$ For example, the largest binding energy of an Au dimer in the vicinity of the 5-8-5 DV is only $\sim 1.2 \mathrm{eV}$, while the trimer has a formation energy of only $\sim 1.7 \mathrm{eV}$.

The above immediately lead us to an equally fundamental feature of the present design: unlike small Au nanoparticles, ${ }^{13}$ the $U_{\text {diss }}$ of the Au dopant in defected graphene- and in general of the entire system-is significantly increased. This again is caused by the strong bonds made by Au with carbon, which yield a binding energy almost $0.7 \mathrm{eV}$ larger than the experimental cohesive energy of bulk Au. Hence, based on the binding energy of the Au dopant and the fact that the dissolution potential of bulk $\mathrm{Au}$ is about $1.5 \mathrm{~V} / \mathrm{SHE}$, the nominal $U_{\text {diss }}$ for the $\mathrm{Au}$ atoms doped in the 5-8-5 DV is predicted to be $\sim 1.72 \mathrm{~V}$, while that of Pt is only $1.18 \mathrm{~V} / \mathrm{SHE}$. Note that this feature not only indicates that voltammetric cycling of the system in an acidic solvent up to a potential of $\sim 1.5 \mathrm{~V} / \mathrm{SHE}$ will result in dissolving of small Au particles without damaging of the Au-doped graphene but also affords a basis for expecting that this material will overcome the problem that dissolution sets in at a much lower electrode potentials than the nominal $U_{\text {diss }}{ }^{9}$

We have seen that the Au dopant makes strong bonds with carbon atoms and such result is expected to make the PLDOS of the Au dopant dramatically different from that of $\mathrm{Au}(111)$, not only, of course, because it is a single atom but also because of electronic hybridization, in the sense that the Au dopant gets a much higher density of states close to $\mathrm{E}_{\mathrm{F}}$. Hence, before turning to our main result, that is, the onset potential of $\mathrm{Au}$ doped defected graphene, we first investigate whether and by which means the rational selection of the material yields the expected enhanced reactivity. Indeed, Figure 6(a) shows that the electronic states of the Au dopant are dramatically different from those of an $\mathrm{Au}(111)$ surface atom. First, it is surprising that, rather than the $d$-states, the $s$ - and $p$-states of gold (which are distributed very broadly in energy for Au surfaces) are those forming the sharpest peaks close to $\mathrm{E}_{\mathrm{F}}$. Second, in contrast to the $d$-states of $\mathrm{Au}(111)$, those of the doped $\mathrm{Au}$ undergo a wide splitting. In fact, both Au- $d_{x y}$ and C- $p_{x}$ states split making a 4-eV gap between occupied and unoccupied states (compare with Fig. 2). The unoccupied states correspond to a high narrow peak at $\sim 5 \mathrm{eV}$ above $\mathrm{E}_{\mathrm{F}}$ that originates from the hybridization of the in-plane $d$-states of Au with the $s p^{2}$-states of its neighboring $\mathrm{C}$ atoms. Explicitly, the sharp peak located at $\sim 5 \mathrm{eV}$ for the in-plane PLDOS of both atoms arises from a strong hybridization between the in-plane-oriented Au- $d_{x y}$ and C- $p_{x}$ states, as seen from Fig. 6(b). Clearly, such significant
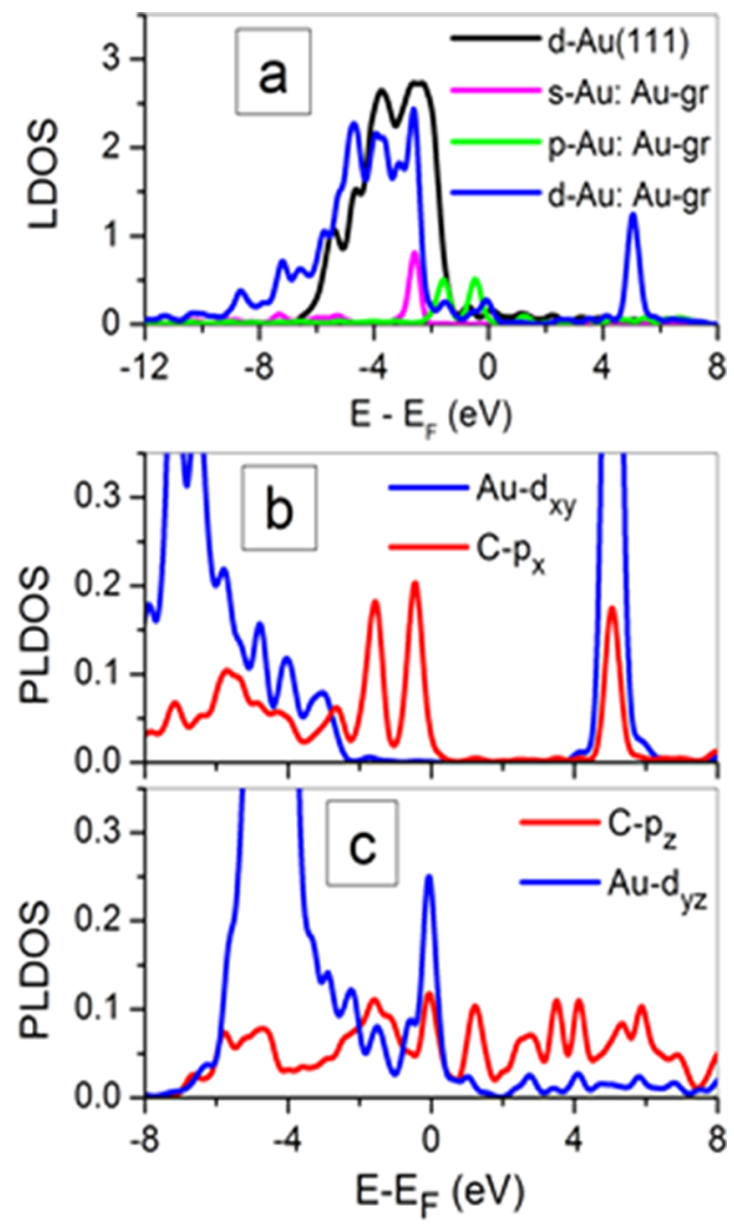

FIG. 6. Panel (a): LDOS of the Au dopant of defected graphene (with 5-8-5 DV in the $4 \times 4$ structure) and the d-LDOS of a surface atom of $\mathrm{Au}(111)$. Panels (b) and (c): selected projected LDOS of the Au dopant of defected graphene (with a 5-8-5 DV) and of its first $\mathrm{C}$ neighbors (type 1). (Top) In-plane: Au-d $\mathrm{d}_{\mathrm{xy}}$ and C- $\mathrm{p}_{\mathrm{x}}$; (bottom) out-of-plane: Au-d $\mathrm{d}_{\mathrm{yz}}$ and C- $\mathrm{p}_{\mathrm{z}}$. 
amount of unoccupied anti-bonding states, formed because of hybridization, reflects the strong $\mathrm{Au}-\mathrm{C}$ bonding that makes the binding energy of dopant Au so high as to increase $U_{\text {diss }}$ so dramatically, as mentioned above. And yet, a third asset of the present design is that there is a number of peaks of both Au- $d_{y z}$ and C- $p_{z}$ out-of-plane PLDOSs with the same energy speaking of a significant hybridization between them. Some of these peaks are around the Fermi level, thus yielding that the $\mathrm{Au}$ dopant and its $\mathrm{C}$ neighbors have a much higher DOS around $\mathrm{E}_{\mathrm{F}}$ than that found in pristine graphene or $\mathrm{Au}(111)$ (see Fig. 6(c)). These peaks in the LDOS of the Au dopant and the edge $\mathrm{C}$ atoms-whose orientation is normal to the graphene sheet plane-are those responsible for the high catalytic activity of Au-doped graphene that we shall detail in the following.

\section{B. Thermodynamics of oxygen reduction reaction on the Au-doped graphene}

We now turn to determine the ORR activity of Au-doped graphene through the ORR free energy diagrams. As described in Sec. II, we express the total energy contributions to the free energy $G$ through binding energies of the ORR intermediates (Eqs. (7), (8), and a similar equation for $\mathrm{OOH}$ ) on the $\mathrm{Au}-$ doped graphene. The binding energies obtained for active adsorption sites are listed in Table I.

One can see from the table that $\mathrm{O}$ prefers to adsorb at the $\mathrm{Au}-\mathrm{C}$ bridges, while $\mathrm{C}$ top sites are preferred for $\mathrm{OH}$ and $\mathrm{OOH}$. The most favorable sites are found to be the $\mathrm{Au}-\mathrm{C} 1$ bridge for $\mathrm{O}$ and the $\mathrm{C} 1$ top for $\mathrm{OH}$ and $\mathrm{OOH}$. We thus calculate the ORR free energies for these sites. Note that the binding energy of the ORR intermediates on Audoped graphene is found to be in the order favorable for the ORR: $E_{B}(O)>E_{B}(O H)>E_{B}(O O H)$-i.e., the same order reported for Pt- and Pd-based ORR catalysts and some other transition metals. ${ }^{7,32,36}$ However, there is a key difference in the binding properties between Au-doped graphene and $\operatorname{Pt}(111)$; for example, $E_{B}(O)$ is $0.24 \mathrm{eV}$ lower for Au-doped graphene than for $\mathrm{Pt}$, and nevertheless $E_{B}(O H)$ is $0.12 \mathrm{eV}$ higher for Au-graphene than for $\mathrm{Pt}(111)$. This finding is conceptually significant, because it shows that the linear dependence between these energies, obtained for Pt- and Pdbased catalysts, ${ }^{34,36}$ does not hold in general. This faulty notion is widely used for combinatorial screening of metal/alloy surfaces as possible candidates for ORR catalysts. In fact, authors often calculate only $E_{B}(O)$ and express free energies of all other ORR intermediates assuming and applying the linear relation in order to handle numerous proposed materials. ${ }^{33,34}$
However, our results demonstrate that the value of $E_{B}(O)$ is not sufficient to evaluate activity of any arbitrary material toward ORR. Part of the reason is that the notion of the bindingenergy linear scaling was developed within a model ${ }^{32}$ that ignores the perturbation undergone by a substrate because of adsorbates. ${ }^{51}$ This perturbation increases the total energy of the system and thus in general decreases the binding energy. ${ }^{51}$ Therefore, this effect is critical for determining the reactivity of a substrate. For instance, since $\mathrm{O}$ binds to $\mathrm{Au}$-graphene stronger than $\mathrm{OH}$, its perturbation energy is higher than that of $\mathrm{OH}$. Consequently, the binding-energy reduction of due to the substrate perturbation should be larger for $\mathrm{O}$ than for $\mathrm{OH}$. Hence, bearing in mind that the linear scaling fails for the system under consideration, here we perform the calculations of the free energies for all ORR intermediates adsorbed on Au-doped graphene using Eq. (6) for both the $4 \times 4$ and $6 \times 6$ structures. To obtain the ORR free energies, we recalculate the binding energies of the ORR intermediates at the presence of water molecules (four molecules per supercell), which simulate the solvent effects. ${ }^{32,33}$ We also calculate the zeropoint energies of the ORR intermediates adsorbed on the preferred sites. The latter are calculated only for the $4 \times 4$ structures, because, as shown for a number of catalysts, ${ }^{36}$ variation of ZPE from one surface to the other is very small. The results are listed in Table II.

We find that the structural optimization rearranges the $\mathrm{H}_{2} \mathrm{O}$ molecules in such a way that a positively charged hydrogen atom of a hydroxyl species turns toward the negatively charged oxygen of water making a hydrogen bond. $\mathrm{OH}$ and $\mathrm{OOH}$ are flexible in the sense that they tilt to contribute to these hydrogen bonds (see Figure 7) while staying bound to their adsorption sites. This effect reduces the total energy of the system and, as a result, increases $E_{B}(O H)$ and $E_{B}(O O H)$, with respect to the values obtained without water (see Table I). Oxygen atoms strongly bound to Au-doped graphene do not have such a degree of freedom, therefore they make weaker hydrogen bonds with water and only slightly disturb the $\mathrm{H}_{2} \mathrm{O}$ order that leads to a minor reduction of $E_{B}(O)$, as seen from Table II. It is worth mentioning that a similar behavior has been reported for other systems. 7,36

Next, for both the $4 \times 4$ and $6 \times 6$ structures, we build the ORR free energy diagram of Au-doped graphene for a zero electrode potential, i.e., $\Delta G_{U}=0$. This reveals that for the $4 \times 4$ structure, the $\mathrm{HO}^{*}+\left(\mathrm{H}^{+}+\mathrm{e}^{-}\right) \rightarrow \mathrm{H}_{2} \mathrm{O}+{ }^{*}$ reaction step is the rate-limiting one for ORR on Au-doped graphene. This step thus determines its onset potential $\left(U_{0}\right)$, which is $0.8 \mathrm{~V} / \mathrm{SHE}$. In turn, for the $6 \times 6$ structure, we find the

TABLE I. Binding energy (in eV) of the ORR intermediates calculated for the active adsorption sites of the $(6 \times 6)$ Au-doped graphene structure. The notation refers to that used in Fig. 1. Notice that in the second column, the binding energies at the preferred sites are provided for both $6 \times 6$ and $4 \times 4$ structures.

\begin{tabular}{|c|c|c|c|c|c|}
\hline Adsorption site & Au-1-bridge & $\begin{array}{c}2-2- \\
\text { bridge }\end{array}$ & $\begin{array}{c}\text { 1-2- } \\
\text { bridge }\end{array}$ & $\begin{array}{c}\text { 1-3- } \\
\text { bridge }\end{array}$ & $\begin{array}{c}\text { 3-4- } \\
\text { bridge }\end{array}$ \\
\hline Adsorbate: $\mathrm{O}$ & $4.288($ for $4 \times 4: 4.293)$ & 3.383 & 3.360 & 3.215 & 2.719 \\
\hline Adsorption site & Top C1 & Top C2 & Top C3 & Top C4 & Top-Au \\
\hline Adsorbate: $\mathrm{OH}$ & $2.395($ for $4 \times 4: 2.561)$ & 1.619 & 1.141 & 1.834 & 0.825 \\
\hline Adsorbate: $\mathrm{OOH}$ & $0.826($ for $4 \times 4: 0.954)$ & 0.047 & Not stable & 0.297 & Not stable \\
\hline
\end{tabular}


TABLE II. Binding energies (in eV) of the ORR intermediates co-adsorbed with water and $\mathrm{ZPE}$ (in eV) on the preferred sites on Au-doped graphene.

\begin{tabular}{lccc}
\hline \hline & $\mathrm{O}$ & $\mathrm{OH}$ & $\mathrm{OOH}$ \\
\hline $\mathrm{E}_{\mathrm{B}}(4 \times 4)$ & 4.233 & 2.790 & 1.103 \\
$\mathrm{E}_{\mathrm{B}}(6 \times 6)$ & 4.228 & 2.625 & 0.976 \\
$\mathrm{ZPE}$ & 0.067 & 0.320 & 0.439 \\
\hline \hline
\end{tabular}

$\mathrm{O}^{*}+\left(\mathrm{H}^{+}+\mathrm{e}^{-}\right) \rightarrow \mathrm{HO}^{*}$ reaction step to be the rate limiting one, yielding an onset potential of $0.67 \mathrm{~V} / \mathrm{SHE}$. Note, as a reference, that using the same approximations, we obtain for $\operatorname{Pt}(111) U_{0}=0.68 \mathrm{~V} / \mathrm{SHE}$, which is in good agreement with previous calculations. ${ }^{32,33}$ We thus conclude that for a low $\mathrm{Au}$ dopant concentration, the Au-graphene system is approximately as active toward ORR as $\mathrm{Pt}$, while for a higher concentration $(4 \times 4)$, the onset potential may be even $0.12 \mathrm{eV}$ higher than that of Pt.

A word of caution is necessary in interpreting the calculated onset potential for Pt. $U_{0}$ is smaller than that obtained from experiment ( $\sim 0.9 \mathrm{~V} / \mathrm{SHE})$ in large part because in experiment $U_{0}$ is measured at very low current, which corresponds to a very low transition rate. Such low rate is thus achievable at $U$ higher than the computed $U_{0}$ because endothermic reaction steps are taking place. The computed $U_{0}$, however, is obtained when the states involved in the ratelimiting reaction step are at equilibrium.

To illustrate the ORR activity of Au-doped graphene and compare it to that of $\operatorname{Pt}(111)$, we build the ORR diagrams for Au-doped graphene for both the $(4 \times 4)$ and $(6 \times 6)$ structures, as well as for $\operatorname{Pt}(111)$ for an electrode potential of $U=0.68 \mathrm{~V}$ (i.e., $U_{0}$ of $\mathrm{Pt}$ ) and plot them together in Figure 8. The figure demonstrates that since $U=U_{0}$ of $\operatorname{Pt}(111)$, the last ORR two states on $\operatorname{Pt}(111)$ are in equilibrium, whereas the $\mathrm{O}^{*}+\left(\mathrm{H}^{+}+\mathrm{e}^{-}\right) \rightarrow \mathrm{HO}^{*}$ step on Au-doped $6 \times 6$ structure is only slightly endothermic $(10 \mathrm{meV})$ and all ORR steps are exothermic for the more active Au-doped $4 \times 4$ structure. Importantly, even though $\mathrm{OOH}$ binds to $\mathrm{Au}$-doped graphene weaker than to $\operatorname{Pt}(111)$, at this electrode potential, it is still bound strong enough to ensure a low rate of its desorption. It is

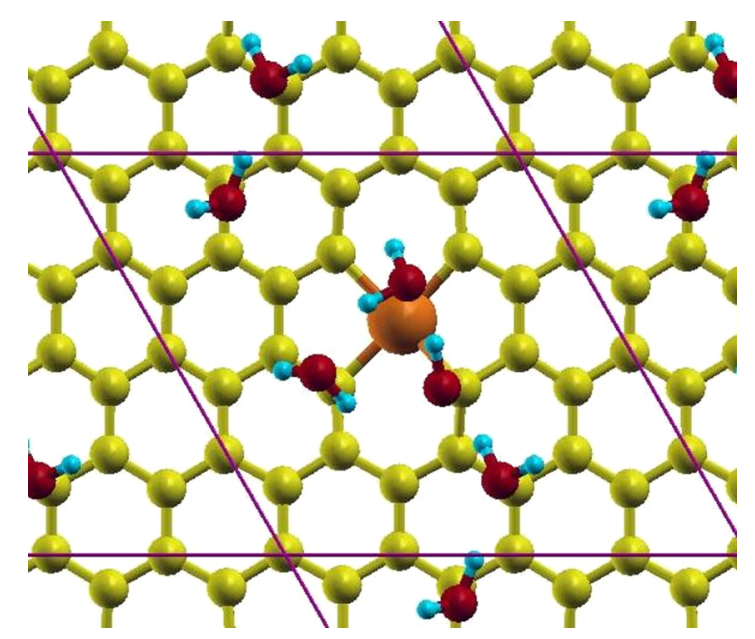

FIG. 7. Geometry of adsorption of $\mathrm{OH}$ and four water molecules on $(4 \times 4)$ $\mathrm{Au}-\mathrm{Gr}$ structure. Red and blue balls represent $\mathrm{O}$ and $\mathrm{H}$ atoms, respectively. Purple lines mark the supercell boundaries.

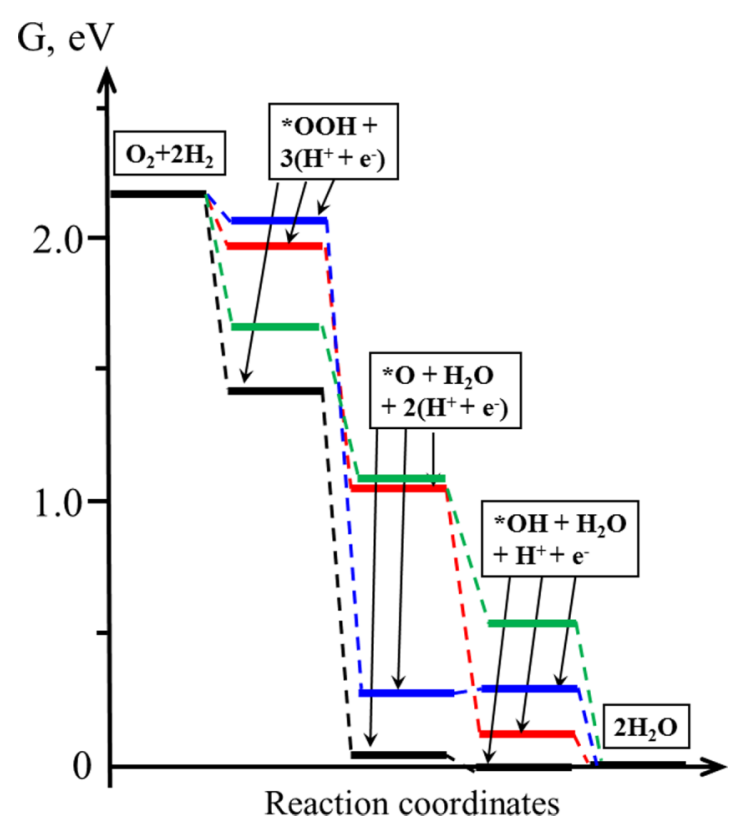

FIG. 8. The ORR diagrams built for Au-graphene $4 \times 4$ and $6 \times 6$ (red and blue lines, respectively), and $\mathrm{Pt}(111)$ (black lines) for the electrode potential $U=0.68 \mathrm{~V} / \mathrm{SHE}$. Green lines represent free energies for an ideal ORR catalyst.

worth to compare the obtained results not only with $\mathrm{Pt}(111)$ but also with defected graphene doped with elements other than gold. Similar calculations performed for N-doped graphene $\mathrm{e}^{23}$ resulted in $U_{0}=0.51 \mathrm{~V} / \mathrm{SHE}$ that is much lower that $U_{0}$ obtained in our work for Au doped graphene. As mentioned in the Introduction, the reduced graphene oxide doped with $\mathrm{FeN}_{4}$ complexes exhibits a high activity toward ORR at a low electrode potential, however its dissolution potential as unacceptably low. ${ }^{21}$ The authors of Ref. 24 estimated the ORR performance of graphene doped with non-noble metals using their computational results on oxygen molecular adsorption. They suggest that graphene doped with $\mathrm{Ni}, \mathrm{Pd}$, and Sn may be highly active toward ORR. However, disadvantage of these materials can be a low resistance against dissolution. Indeed, bulk $\mathrm{Ni}$, for example, has $U_{\text {diss }}=-0.26 \mathrm{~V} / \mathrm{SHE}$. Therefore, in order to match the $U_{\text {diss }}$ of Pt, $0.9 \mathrm{~V}$, the $U_{\text {diss }}$ of $\mathrm{Ni}$ has to be increased by $1.16 \mathrm{~V}$. Using the models provided in Refs. 10 and 13, we estimate that in order to reach $U_{\text {diss }}=0.9 \mathrm{~V}$ for $\mathrm{Ni}$, the $\mathrm{Ni}-\mathrm{C}$ bonds have to be at least $2.32 \mathrm{eV}$ stronger than $\mathrm{Ni}-\mathrm{Ni}$ bonds in bulk $\mathrm{Ni}$, which is hard to expect. Thus, the main advantage of Au-doped graphene over the systems, discussed above, however, is its unprecedentedly high electrochemical resistance. Combined with pretty high activity toward ORR, this property makes the proposed material a promising electrocatalyst for ORR in hydrogen fuel cell cathodes.

Remarkably, had we applied the above-mentioned linear scaling approximation ${ }^{33,34}$ instead of the actual calculation of the entire free-energy diagram, we would have incorrectly predicted that the free energy of the $* \mathrm{OH}$ state on Au-doped graphene is much higher than it actually is. Consequently, the separation between free energies of the $* \mathrm{O}$ and $* \mathrm{OH}$ states would have been predicted-incorrectly again - to be too small and so would $U_{0}$ as well. In other words, had we 
applied that widely used linear approximation, we would have missed the Au-graphene as a promising ORR catalyst.

\section{CONCLUSIONS}

With the goal of designing an electrocatalyst that is highly active toward ORR and stable in reaction environment, here we propose gold-doped graphene sheets as electrocatalysts for the ORR in hydrogen fuel cell cathodes. Our density-functionaltheory-based calculations show that $\mathrm{Au}$ atoms incorporated into the 5-8-5 carbon di-vacancies of graphene make strong covalent bonds with four neighboring $\mathrm{C}$ atoms, such that the Au binding energy is $0.43-0.67 \mathrm{eV}$ higher than the bulk $\mathrm{Au}$ cohesive energy, depending on the concentration of 5-8-5 DV. Importantly, such strong binding of the Au dopant ensures high stability of the system against dissolution in the reaction environment, even higher than that of the bulk $\mathrm{Au}$ surfaces. On the other hand, the formation energy of $\mathrm{Au}$ clusters anchored to the Au dopants is relatively weak compared to the binding energy of the latter, which makes easy their removal during preparation. The significant hybridization between the electronic states of the dopant $\mathrm{Au}$ atom and its four $\mathrm{C}$ neighbors passivates but simultaneously activates the latter $\mathrm{C}$ sites for the ORR. We find that the significant hybridization between Au-dand C-p-states (that is also responsible for the high binding energy of the doped $\mathrm{Au}$ ) brings a substantial amount of these states to the Fermi-level. As a result, the $\mathrm{C}$ atoms bound to Au become active adsorption sites for the ORR intermediates, as indicated by our calculations of the binding energies of $\mathrm{O}, \mathrm{OH}$, and $\mathrm{OOH}$. We calculate free energies of the ORR intermediates adsorbed on the Au-doped graphene to build the reaction free energy diagrams. Analysis of the ORR free energy diagrams based on the above-mentioned calculations brings us to the conclusion that the Au-doped graphene proposed in this work is more or as active toward ORR as Pt and much more stable against dissolution than the bestknown Pt-based electrocatalysts. Remarkably, the comparison between the binding energy of the ORR intermediates on the Au-doped graphene and those on $\operatorname{Pt}(111)$ shows that the widely accepted notion of a linear scaling between $E_{B}(O)$, $E_{B}(\mathrm{OH})$, and $E_{B}(\mathrm{OOH})$ does not hold in this case and thus it may not be transferable to systems other than some metal surfaces. The significance of this result is that $E_{B}(O)$ alone is not sufficient to evaluate the activity of a material toward ORR. Therefore, in contrast to a number of authors, who express the entire ORR free energy diagram through $E_{B}(O)$ only, here we perform complete calculations of the free energies of all ORR intermediate and final states.

${ }^{1}$ C. Wang, D. van der Vliet, K. L. More, N. J. Zaluzec, S. Peng, S. Sun, H. Daimon, G. Wang, J. Greeley, J. Pearson et al., Nano Lett. 11, 919 (2011).

${ }^{2}$ R. R. Adzic, J. Zhang, K. Sasaki, M. B. Vukmirovic, M. Shao, J. X. Wang, A. U. Nilekar, M. Mavrikakis, J. A. Valerio, and F. Uribe, Top. Catal. 46, 249 (2007).

${ }^{3}$ J. Zhang, M. B. Vukmirovic, Y. Xu, M. Mavrikakis, and R. R. Adzic, Angew. Chem., Int. Ed. 44, 2132 (2005).

${ }^{4}$ A. Nilekar and M. Mavrikakis, Surf. Sci. 602, L89 (2008).

${ }^{5}$ W.-P. Zhou, X. Yang, M. B. Vukmirovic, B. E. Koel, J. Jiao, G. Peng, M. Mavrikakis, and R. R. Adzic, J. Am. Chem. Soc. 131, 12755 (2009).

${ }^{6}$ K. Gong, W.-F. Chen, K. Sasaki, D. Su, M. B. Vukmirovic, W. Zhou, E. L. Izzo, C. Perez-Acosta, P. Hirunsit, P. B. Balbuena, and R. R. Adzic, J. Electroanal. Chem. 649, 232 (2010).
${ }^{7}$ S. Stolbov and M. A. Ortigoza, J. Phys. Chem. Lett. 3, 463 (2012).

${ }^{8}$ D. R. Lide, CRC Handbook of Chemistry and Physics (CRC Press, New York, 1996).

${ }^{9}$ K. Sasaki, M. Shao, and R. R. Adzic, "Dissolution and stabilization of platinum in oxide cathodes," in Polymer Electrolyte Fuel Cell Durability, edited by F. N. Büchi, M. Inaba, and T. J. Schmidt (Springer, 2009).

${ }^{10}$ J. Greeley and J. K. Nørskov, Electrochim. Acta 52, 5829 (2007).

${ }^{11}$ K. Sasaki, H. Naohara, Y. Cai, Y. M. Choi, P. Liu, M. B. Vukmirovic, X. Jia, J. X. Wang, and R. R. Adzic, Angew. Chem., Int. Ed. 49, 8602 (2010).

${ }^{12}$ M. Haruta, Catal. Today 36, 153 (1997).

${ }^{13}$ R. Jinnouchi, E. Toyoda, T. Hatanaka, and Y. Morimoto, J. Phys. Chem. C 114, 17557 (2010).

${ }^{14}$ S. Guo and S. Sun, J. Am. Chem. Soc. 134, 2492 (2012).

${ }^{15}$ Y. Liang, Y. Li, H. Wang, J. Zhou, J. Wang, T. Regier, and H. Dai, Nat. Mater. 10, 780 (2011).

${ }^{16}$ H. Yin, H. Tang, D. Wang, Y. Gao, and Z. Tang, ACS Nano 6, 8288 (2012).

${ }^{17}$ J.-J. Lv, S.-S. Li, A.-J. Wang, L.-P. Mei, J.-R. Chen, and J.-J. Feng, Electrochim. Acta 136, 521 (2014)

${ }^{18}$ F.-B. Wang, J. Wang, L. Shao, Y. Zhao, and X.-H. Xia, Electrochem. Commun. 38, 82 (2014).

${ }^{19}$ L. Zhang and Z. Xia, J. Phys. Chem. C 115, 11170 (2011).

${ }^{20}$ Y. Jiao, Y. Zheng, M. Jaroniec, and S. Z. Qiao, J. Am. Chem. Soc. 136, 4394 (2014).

${ }^{21}$ H. R. Byon, J. Suntivich, and Y. Shao-Horn, Chem. Mater. 23, 3421 (2011).

${ }^{22}$ S. Kattel and G. Wang, J. Phys. Chem. Lett. 5, 452 (2014).

${ }^{23}$ F. Studt, Catal. Lett. 143, 58 (2013).

${ }^{24}$ M. Kaukonen, A. V. Krasheninnikov, E. Kauppinen, and R. M. Nieminen, ACS Catal. 3, 159 (2013)

${ }^{25}$ W. Liang, J. Chen, Y. Liu, and S. Chen, ACS Catal. 4, 4170 (2014)

${ }^{26} \mathrm{C}$. Zhu and S. Dong, Nanoscale 5, 1753 (2013).

${ }^{27}$ F. Banhart, J. Kotakoski, and A. V. Krasheninnikov, ACS Nano 5, 26 (2011).

${ }^{28}$ O. Cretu, A. V. Krasheninnikov, J. A. Rodriguez-Manzo, L. Sun, R. M. Nieminen, and F. Banhart, Phys. Rev. Lett. 105, 196102 (2010).

${ }^{29}$ A. V. Krasheninnikov and R. M. Nieminen, Theor. Chem. Acc. 129, 625 (2011).

${ }^{30}$ S. Malola, H. Häkkinen, and P. Koskinen, Appl. Phys. Lett. 94, 043106 (2009).

${ }^{31}$ W. Zhang, L. Sun, Z. Xu, A. V. Krasheninnikov, P. Huai, Z. Zhu, and F. Banhart, Phys. Rev. B 81, 125425 (2010).

${ }^{32}$ J. K. Nørskov, J. Rossmeisl, A. Logadottir, L. Lindqvist, J. R. Kitchin, T. Bligaard, and J. Jónsson, J. Phys. Chem. B 108, 17886 (2004).

${ }^{33}$ J. Greeley, I. E. L. Stephens, A. S. Bondarenko, T. P. Johansson, H. A. Hansen, T. F. Jaramillo, J. Rossmeisl, I. Chorkendorff, and J. K. Nørskov, Nat. Chem. 1, 552 (2009).

${ }^{34}$ J. Greeley and J. K. Nørskov, J. Phys. Chem. C 113, 4932 (2009).

${ }^{35}$ V. Stamenkovic, B. Mun, K. J. J. Mayrhofer, P. N. Ross, N. M. Markovic, J. Rossmeisl, J. Greeley, and J. K. Nørskov, Angew. Chem., Int. Ed. 45, 2897 (2006).

${ }^{36}$ S. Zuluaga and S. Stolbov, J. Chem. Phys. 135, 134702 (2011).

${ }^{37}$ F. Abild-Pedersen, J. Greeley, F. Studt, J. Rossmeisl, T. R. Munter, P. G. Moses, E. Skúlason, T. Bligaard, and J. K. Nørskov, Phys. Rev. Lett. 99, 016105 (2007)

${ }^{38}$ J. D. Cox, D. D. Wagman, and V. A. Medvedev, CODATA Key Values for Thermodynamics (Hemisphere Publishing, Corp., New York, 1989).

${ }^{39} \mathrm{G}$. Kresse and J. Furthmüller, Comput. Mater. Sci. 6, 15 (1996).

${ }^{40} \mathrm{G}$. Kresse and J. Joubert, Phys. Rev. B 59, 1758 (1999).

${ }^{41}$ J. P. Perdew, S. Burke, and M. Ernzerhof, Phys. Rev. Lett. 77, 3865 (1996).

${ }^{42}$ A. Kokalj, Comput. Mater. Sci. 28, 155 (2003).

${ }^{43}$ A. V. Krasheninnikov, P. O. Lehtinen, A. S. Foster, P. Pyykkö, and R. M. Nieminen, Phys. Rev. Lett. 102, 126807 (2009).

${ }^{44}$ Y. Zhang, S. Talapatra, S. Kar, R. Vajtai, S. K. Nayak, and P. M. Ajayan, Phys. Rev. Lett. 99, 107201 (2007).

${ }^{45}$ R. G. Amorim, A. Fazzio, A. Antonelli, F. D. Novaes, and A. J. R. da Silva, Nano Lett. 7, 2459 (2007).

${ }^{46}$ G. Blyholder, J. Phys. Chem. 68, 2772 (1964).

${ }^{47}$ S.-S. Sung and R. Hoffmann, "How carbon monoxide bonds to metal surfaces," J. Am. Chem. Soc. 107, 578 (1985).

${ }^{48}$ Y. Gan, L. Sun, and F. Banhart, Small 4, 587 (2008).

${ }^{49}$ S. Koh and P. Strasser, J. Am. Chem. Soc. 129, 12624 (2007).

${ }^{50}$ P. Strasser, S. Koha, and J. Greeley, Phys. Chem. Chem. Phys. 10, 3670 (2008).

${ }^{51}$ M. Alcántara Ortigoza and S. Stolbov, "The perturbation energy: A missing key to understand "nobleness" of bulk gold," J. Chem. Phys. (submitted). 